(C) [2008] IEEE. Reprinted, with permission, from Jin Jianxun., Chen Xiaoyuan.,Guo Youguang., Zhan Yuedong and Zhu Jianguo. Proceedings of the $27^{\text {th }}$ Chinese Control Conference July 16-18, 2008, Kunming,Yunnan, China. This material is posted here with permission of the IEEE. Such permission of the IEEE does not in any way imply IEEE endorsement of any of the University of Technology, Sydney's products or services. Internal or personal use of this material is permitted. However, permission to reprint/republish this material for advertising or promotional purposes or for creating new collective works for resale or redistribution must be obtained from the IEEE by writing to pubs-permissions@ieee.org. By choosing to view this document, you agree to all provisions of the copyright laws protecting it 


\title{
HTS Transformer and Its Related Loss Measurement and Quench Protection Techniques
}

\author{
Jianxun $\mathrm{Jin}^{1}$, Xiaoyuan $\mathrm{Chen}^{1}$, Youguang Guo ${ }^{2}$, Yuedong Zhan ${ }^{2,3}$, Jianguo Zhu \\ 1. Center of Applied Superconductivity and Electrical Engineering, \\ University of Electronic Science and Technology of China, Chengdu, China 610054 \\ E-mail: jxjin@uestc.edu.cn cxy_yjs@yahoo.com.cn \\ 2. Faculty of Engineering, University of Technology, Sydney, NSW 2007, Australia \\ E-mail: youguang@eng.uts.edu.au joe@eng.uts.edu.au.au \\ 3. Kunming University of Science and Technology, Kunming, China 150001 \\ E-mail: yuedong@eng.uts.edu.au
}

\begin{abstract}
Recently research and development concerning applications of high temperature superconducting (HTS) transformers have been progressed actively in the world. Besides the HTS rated current capability, it is also essential to develop the relevant and precise performance test and protection systems for HTS transformers. This paper mainly presents elementary test and protection methods of HTS transformers including AC loss measurement, and quench detection and protection. A new integrated measurement system for HTS transformers is also proposed by using the Labview virtual instrument software.
\end{abstract}

Key Words: High Temperature Superconductor (HTS), HTS Transformer, AC Loss, Quench Protection, Labview

\section{INTRODUCTION}

With the discovery of HTS with higher critical temperature, electrical applications of HTS become more attractive than conventional low temperature superconductor (LTS), due to operation cost reduced dramatically. The HTS transformer has been expected to be one of the most promising practical applications, and the advantages of HTS transformers compared to normal transformers have been well described [1, 2], high efficiency and substantial energy saving have been readily identified.

Researches on the applications of HTS transformers have been progressed actively in the world [3, 4]. In USA, a three-phase 5/10 MVA $(24.9 \mathrm{kV} / 4.2 \mathrm{kV})$ HTS power transformer has been manufactured since 2004 [5]. In Japan, a single-phase 2 MVA $(66 \mathrm{kV} / 6.9 \mathrm{kV})$ HTS power transformer has been developed since 2004 [6]. Siemens in Germany has developed a single-phase 1 MVA (25kV/ $1.4 \mathrm{kV}$ ) traction transformer since 2001 [7], with an aim to apply in high-speed rail system. In 2004, Railway Technical Research Institute in Japan completed a single-phase 4 MVA $(25 \mathrm{kV} / 1.2 \mathrm{kV})$ HTS traction transformer for Shinkansen rolling stock [8].

Unlike conventional ones, HTS transformers have different electromagnetic properties. HTS windings usually have three superconducting critical values: critical temperature, critical magnetic field, and critical current density. HTS transformers must be operated within the limit of those three values. Otherwise, quench occurs and thus affects the performance of the HTS transformers. So, it is necessary to detect superconducting critical values in advance and protect HTS transformers effectively when quench occurs. It is also important to detect total AC losses in HTS transformers and thus verify the possible commercial advantages.

This paper presents elementary test and control methods of HTS transformers including AC loss measurement methods, and quench detection and protection methods. A new measurement system for HTS transformers is also proposed by using the Labview virtual instrument software.

\section{AC LOSS MEASUREMENT METHODS}

It is essential to test AC losses accurately for evaluation of HTS transformer performance. Electrical method is generally used to measure AC loss of HTS conductors. Although this method has a high sensitivity and a wide range, the measured values can not be well verified yet. Calorimetric methods have rather low sensitivity, require a complicated device and a long time to reach thermal equilibrium, but it can directly measure all the losses including AC loss.

For electrical method, AC four-terminal method is frequently used to evaluate the HTS conductor AC losses. Four different calorimetric measurement methods are examined: measurement of the amount of $\mathrm{LN}_{2}$ evaporation, the temperature rise of the HTS conductor, the temperature difference in flowing $\mathrm{LN}_{2}$, and the $\mathrm{LN}_{2}$ level in the cryostat.

\subsection{AC Four-Terminal Method}

Usually, current signal is large but voltage is very feeble in a HTS conductor because it commonly operates at high current and low resistance situation. It is very difficult to measure AC loss by voltage signal that should be separated from noise because AC loss does not have correct sine waveform due to the nonlinear characteristics. The most popular method for reducing the noise is to use a Lock-in 
amplifier. The reference phase of the applied current is derived by a non-inductive resistor which is in series with the HTS conductor. AC loss is measured by the Lock-in amplifier and a cancel coil is used for the noise reduction. Figure 2 shows this AC loss measurement system [9].

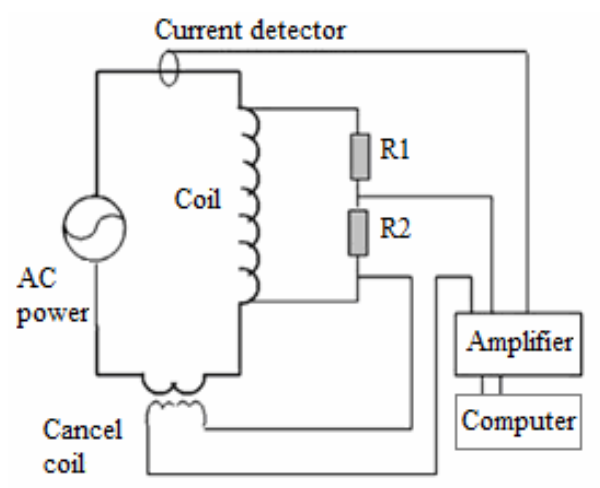

Fig 2. AC loss measurement system.

According to Figure 2, the current (I) of HTS coil, the HTS coil voltage $(\mathrm{V})$, and the phase difference $(\theta)$ between the current and voltage are measured by the Lock-in amplifier, thus AC loss values can be calculated by $I \times V \times \cos \theta$.

\subsection{Measurement of Conductor Temperature}

The essence of this technique is to measure a small temperature increase at a local point in a HTS conductor. The temperature rise is measured by using a differential thermocouple (DTC). The length of the HTS conductor involved can be minimized and consequently the induced noise in the thermocouple circuit is also minimized. The thermocouples are insulated with Teflon. DTC response, which can be subsumed a calibrated constant, is several $\mu \mathrm{V}$ for every $1 \mathrm{~K}$ difference in temperature between the active and reference junctions.

In order to produce a measurable temperature increase, the area of interest must be thermally insulated from $\mathrm{LN}_{2}$. This is done using two pieces of Styrofoam to "sandwich" the HTS conductor or tape, ingress of liquid to this sandwich is prevented by silicon rubber sealant around the tape. This produces a bond with good thermal conductivity but electrically isolated. Electrical isolation is necessary because of the relatively large common mode voltage which may appear on one junction of the thermocouple and it is also important to use as little sealant as possible since this material will reduce the sensitivity of calorimeter at low temperature. The whole is usually immersed in $\mathrm{LN}_{2}$ under atmospheric pressure, and the thermocouple is thereby referenced to a very stable temperature point. Figure 3 shows a schematic of the calorimeter arrangement and placement of the DTC [10].

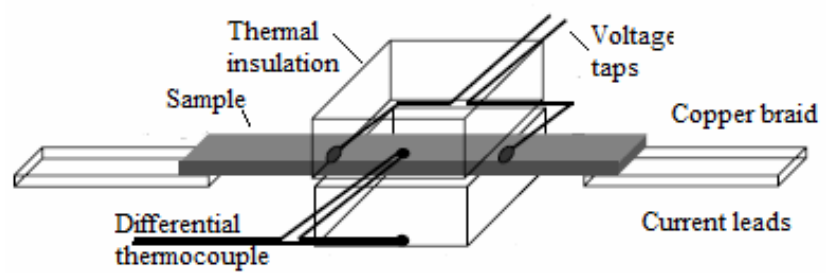

Fig 3. Sketch of measurement of conductor temperature.

\subsection{Measurement of Temperature Difference of $\mathbf{L N}_{2}$}

The method of measuring temperature difference is considered as one of the most stable measurements. Because it is based on the simple measurement and can verify that the AC loss was dependent on the temperature and the flow rate of $\mathrm{LN}_{2}$. The calorimetric device based on this architecture is shown in Figure 4 [11]. This calorimetric device has the low heat in-leakage and high-resolution thermometers and it can evaluate AC loss accurately even though the HTS conductor length is short.

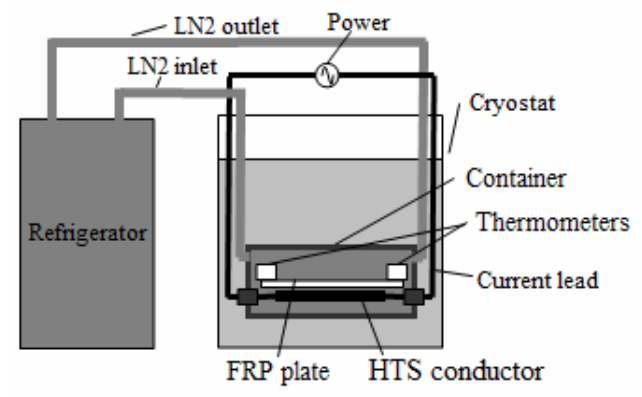

Fig 4. Sketch of measurement of $\mathrm{LN}_{2}$ temperature.

The calorimetric container consists of a double-walled pipe. The inner and outer pipes are thermally insulated from each other. The HTS layers are soldered with cylindrical copper at both ends and connected with the current lead in the calorimetric container through the flexible copper conductor. $\mathrm{LN}_{2}$ in the calorimetric container make the closed circulation loop as follows. $\mathrm{LN}_{2}$ travels through the inlet from a circulation pump of refrigerator to container then returns to refrigerator through the outlet. Platinum thermometers are selected to obtain steady repeatability and high resolutions, and are placed on the FRP plate that is placed on the side of HTS conductor [11]. In addition, $\mathrm{LN}_{2}$ in the cryostat reduces the influence of heat generated by the current leads, with an aim to process a precise measurement of AC loss.

\subsection{Measurement of $\mathrm{LN}_{2}$ Evaporation Amount}

A suitable technique which can accurately measure the total losses in a HTS conductor immersed in critical $\mathrm{LN}_{2}$ is the mass boil off technique [12]. A schematic drawing of measurement device is shown in Figure 5.

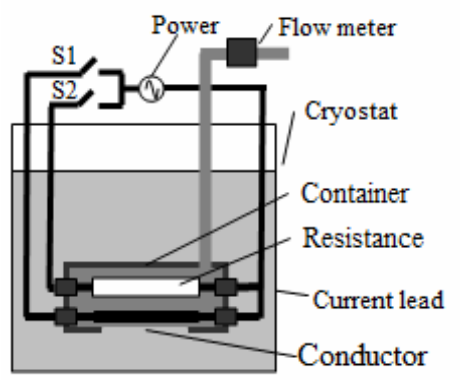

Fig 5. Sketch of measurement of $\mathrm{LN}_{2}$ evaporation amount.

Basically, by sealing the cryogenic vessel which holds the HTS conductor and measuring the gas flow which is vaporized very accurately, the total losses in HTS conductor may be determined by flow meter. The 
measurement steps are presented as follows. Firstly, the value of the background flow should be measured. Secondly, turn on switch $\mathrm{S}_{2}$, ohmic losses in the calibrated resistance can be calculated accurately and detected by flow meter. Then a curve with regard to the ohmic losses and output of flow meter can be drawn as a calibrated curve. At last, turn off switch $S_{2}$ and then turn on switch $S_{1}$, the precise AC loss can be easily obtain by referring to the calibrated curve.

\subsection{Measurement of $\mathrm{LN}_{2}$ Level}

The losses may be measured calorimetrically by monitoring the level of $\mathrm{LN}_{2}$ in the cryostat in which the transformer windings is immersed. Czech Republic has developed a measurement method by using a capacitive meter [13]. A schematic drawing is shown in Figure 6.

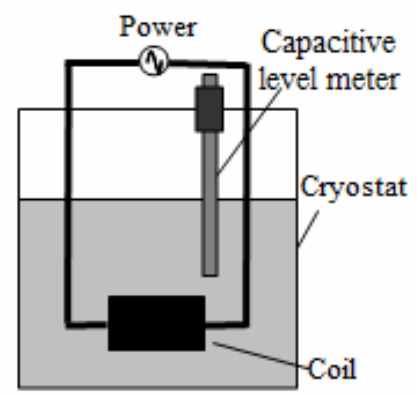

Fig 6. Sketch of measurement of $\mathrm{LN}_{2}$ level.

This capacitive meter consists of three thin wall stainless coaxial tubes with diameter 2, 6, and $10 \mathrm{~mm}$. These tubes are centered using Teflon spacers. The inner tubes form the capacitor, while the outer tube provides electrostatic shielding. The capacitance bridge is used to measure the capacity with relative resolution of $10 \mathrm{ppm}$. As the dielectric constants of the liquid and gaseous nitrogen are different by a factor of 1.43 , the capacity depends on the $\mathrm{LN}_{2}$ level. So, the level of $\mathrm{LN}_{2}$ in the cryostat could be measured using a capacitive level meter.

\section{QUENCH PROTECTION}

When applied alternating currents go up to the critical current of HTS coil, a resistance growth of HTS coil is not so fast. So, even though quench occurs in the HTS coil, a noticeable event is not supposed to be observed. However, there could be several problems within the HTS coil with over current. Local and excessive joule heating may give damage to the HTS coil. So, quench should be detected quickly and precisely so that the winding can be safely discharged. Up to now, there are many widespread technologies for detecting the occurrence of quench, such as resistive voltage measurement method, active power method and AE sensor method.

\subsection{Resistive Voltage Measurement Method}

In this method, two voltage taps are installed on the center of HTS coils (central node 1) and center of three resistances (central node 2). An amplifier is used to amplify voltage difference between central node 1 and 2, then its output signal compares with reference voltage $V_{\text {ref }}$ (criterion of quench occurrence). A practical measurement circuit is shown in Figure 7 [14].

Usually all the coils stay at superconducting states, voltage difference is close to zero and quench signal is low-voltage level accordingly. But if quench occurs, voltage difference increases sharply and output voltage of amplifier is very large, reaches or exceeds $\mathrm{V}_{\text {ref. }}$ So, quench signal voltage becomes high-voltage level and indicates quench occurrence.

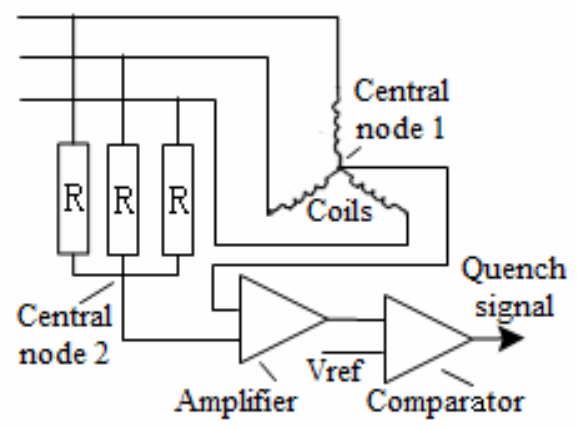

Fig 7. Resistive voltage measurement.

\subsection{Active Power Method}

For resistive voltage measurement method, it is vulnerable to an electromagnetic noise which causes insufficient quench detection and at least needs a central voltage tap in HTS coil. In a large HTS coil, a lead-wire from the central voltage tap may cause a short circuit when high voltage is applied. Japan has developed a quench protection system based on the active power method which detects a quench by measuring the instantaneous active power generated in HTS coil [15]. The protection system is very strong against to the noise and no more needs a central voltage tap.

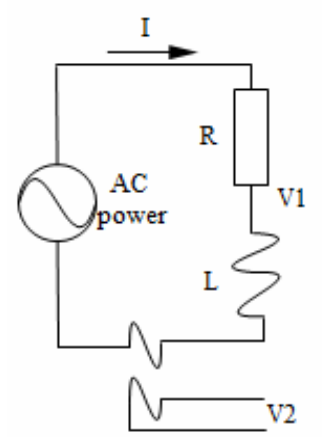

Fig 8. An electric circuit for active power method [15].

As is shown in Figure 8, $\mathrm{V}_{1}$ and $\mathrm{V}_{2}$ are the voltages across a HTS coil and a Rogowski coil, respectively. These voltages are shown as following equations:

$$
V_{1}=R \times I+L \frac{d I}{d t}, \quad V_{2}=M \frac{d I}{d t}
$$

where $R$ is the resistance of the HTS coil and is zero in the superconducting state, $L$ is an inductance of the HTS coil, $I$ is a transport current and $M$ is a mutual inductance of the Rogowski coil. From these equations, an active power $P$ is calculated as follows:

$$
P=\left(V_{1}-\frac{L}{M} V_{2}\right) \times I=R \times I^{2}
$$


In the superconducting state, $P$ is zero and whereas in the normal conductor state it is not zero. Therefore the quench can be detected by measuring $P$.

In this method, low-pass filter (LPF) can eliminate the electromagnetic noise because $P$ has a DC resistive component. So, a quench signal can be detected regardless of the noise. And it is less susceptive to a high voltage, because it is electrically insulated from the HTS coil except both terminal taps. Figure 9 shows a block diagram of the active power method. In this figure, when $P$ becomes larger than a specified threshold, a quench detector recognizes the quench occurrence. The quench detector fully consists of analog circuits such as operation amplifiers and therefore time delay of the detection is very short.

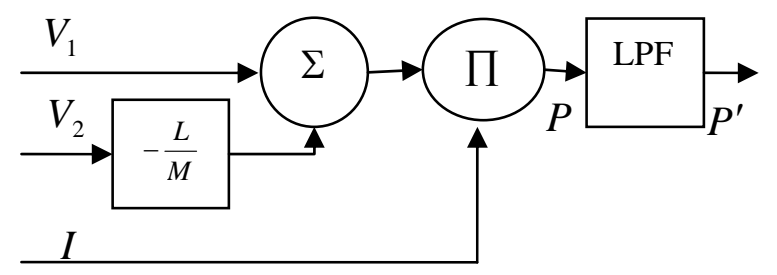

Fig 9. A block diagram of active power method [15].

\subsection{AE Sensor Method}

A large amount of research involving the fields of acoustic emission (AE) has been performed since the late 1970s. Using an AE sensor has proven to be the most successful way to detect mechanical noises from the high performance magnets. This mechanical noise could be generated by the micro crack of epoxy as well as the motion of a conductor from quench. Research has been conducted with regard to the detection of a critical criterion for a HTS transformer winding in AC operation using an AE sensor [16].

It is difficult to detect a resistive voltage by quench in an AC operation, because the major component of the voltage from the winding is inductive in electrical measurement. But AE sensor would be able to detect any mechanical events from quench and any unstable condition of HTS winding. Figure 10 shows a block diagram of acoustic emission data acquisition system [16]. An acoustic emission signal was saved by using an analog data recorder after being amplified by a pre-amplifier and an isolation amplifier.

\subsection{Quench Protection Circuit}

Local and excessive joule heating may give damage to the HTS coils when a quench occurs. So, it is essential that the quench should be detected quickly and precisely so that the coils can be safely discharged. Figure 11 shows a quench protection circuit, which consists of two IGBT switches, a quench detection circuit and a protection resistor [15].

IGBT switches are applicable for both of the direct and alternative transport current. In the superconducting state, IGBT gate signals $G_{1}$ and $G_{2}$ turn on and off, respectively. Then the switch 1 turns on and switch 2 turns off, power source can provide the current $i_{1}$ to the HTS coil. Whereas the quench occurs, $G_{1}$ and $G_{2}$ turn off and on, respectively. $G_{2}$ has to turn on earlier than $G_{1}$ turns off, in order to prevent an excess voltage occurrence in IGBTs. Then the current $i_{1}$ is cut-off and the magnetic energy stored in the coil is dissipated in the protection resistor and excessive joule heating is prevented.

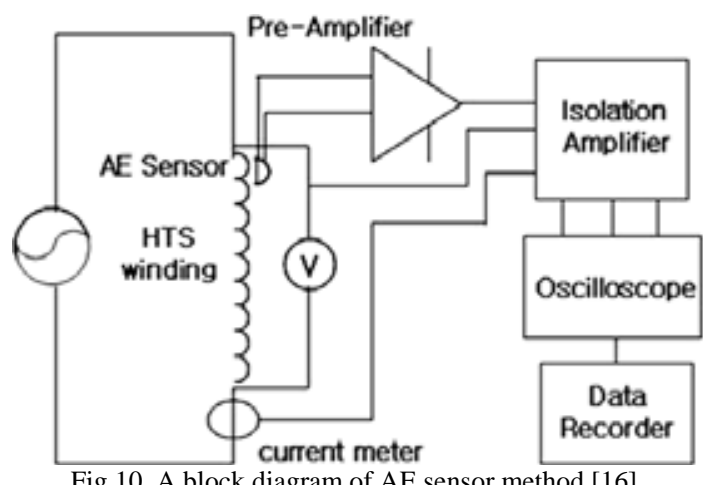

Fig 10. A block diagram of AE sensor method [16].

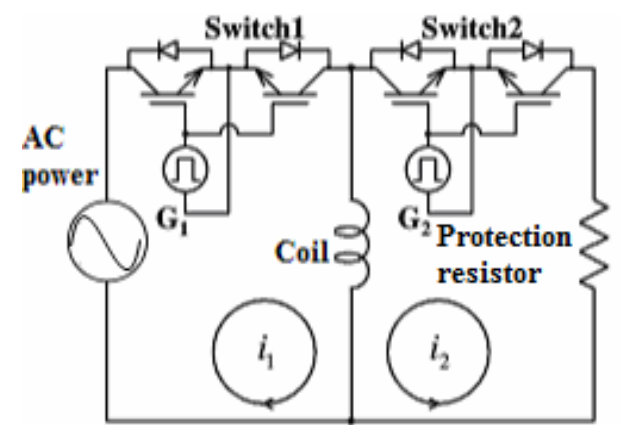

Fig 11. A block diagram of quench protection circuit [15].

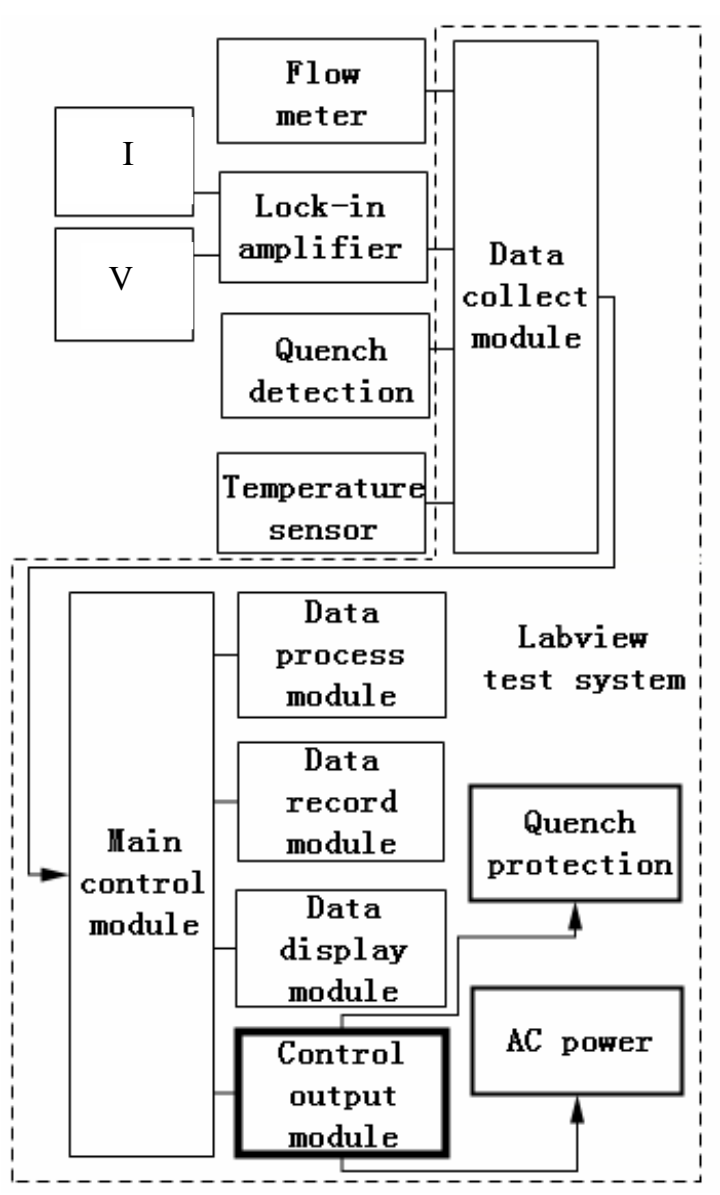

Fig 12. A block diagram of Labview test system. 


\section{LABVIEW TEST SYSTEM DESIGN}

Labview is a graphical programming language based on the virtual instrument software, widely used in the field of measurement and control system. It provides almost the entire classic signal processing function and plenty of advanced signal analysis tools, not only can be easily integrated with hardware to achieve various data acquisition, but also can be used to communicate with a variety of industrial field-bus and thus control of the instruments to achieve more accurate data access, analysis and processing.

The current testing systems for HTS transformers can be solely used to test a characteristic of HTS transformer and lack of the ability to measure a variety of specifications and performance. By using Labview, a new system to achieve accurate and precise performance measurements of HTS transformers is proposed. The proposed test system is the integration of a variety of measurements and can be used to test more comprehensive performance for HTS transformers, such as I-V characteristics test, AC losses test, critical current test, short circuit test, and so on. A block diagram of the Labview test system is shown in Figure 12.

To measure more precise data based on different conditions, we propose a controllable AC power source that can provide adjustable current output, adjustable frequent output and arbitrary signal wave including sine, triangle and square wave. In addition, using Labview, we can achieve quench protection quickly and precisely. A sketch of controllable AC power and quench protection is shown in Figure 13.

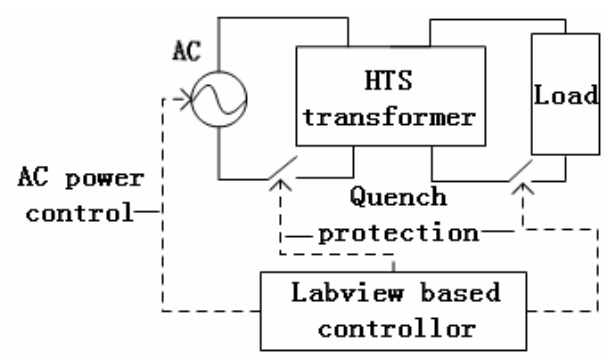

Fig 13. Sketch of controllable power and quench protection.

\section{CONCLUSION}

After the discovery of HTS and the availability of the first HTS wires for use in windings, various groups around the world began working on HTS transformers and recently the focus on the applied superconducting technology for the HTS transformer development has been moving to the industrial preparations from laboratory research stage, and the technology has been well verified for practical applications from small to large scales. It is essential to develop more precise test and control systems for the HTS transformer operation, as the main techniques are summarized in the paper. A new test and control system based on the Labview virtual instrument software has been designed with a conceptual block diagram presented, which provides a method to control and operate HTS transformer more effectively.

\section{REFERENCES}

[1] S. Mehta, N. Aversa, and M. S. Walker, Transforming transformers, IEEE Spectrum, 43-49, 1997.

[2] B. W. McConnell, S. P. Metha, and M. S. Walker, HTS transformers, IEEE Power Engineering Review, Vol.20, No.6, 7-11, 2000.

[3] X. Y. Chen, and J. X. Jin, Development and technology of HTS transformers, Research Communication, Vol.1, No.1, 2007.

[4] X. Y. Chen, and J. X. Jin, Review of HTS transformers and their practical loss measurement and operation protection, Nature Sciences, Vol.2, No.1, 13-23, 2007.

[5] C. S. Weber, C. T. Reis, D. W. Hazelton, S. W. Schwenterly, M. J. Cole, J. A. Demko, E. F. Pleva, S. Mehta, T. Golner, and N. Aversa, Design and operational testing of a 5/10-MVA HTS utility power transformer, IEEE Trans. Appl. Supercond., Vol.15, No.2, 2210-2213, 2005.

[6] T. Bohno, A. Tomioka, M. Imaizumi, Y. Sanuki, T. Yamamoto, Y. Yasukawa, H. Ono, Y. Yagi, and K. Iwadate, Development of $66 \mathrm{kV} / 6.9 \mathrm{kV} 2 \mathrm{MVA}$ prototype HTS power transformer, Physica C, Vol.426-431, 1402-1407, 2005.

[7] R. Schlosser, H. Schmidt, M. Leghissa, and M. Meinert, Development of high-temperature superconducting transformers for railway applications, IEEE Trans. Appl. Supercond., Vol.13, No.2, 2325-2330, 2003.

[8] H. Kamijo, H. Hata, H. Fujimoto, A. Inoue, K. Nagashima, K. Ikeda, M. Iwakuma, K. Funaki, Y. Sanuki, A. Tomioka, H. Yamada, K. Uwamori, and S. Yoshida, Tests of superconducting traction transformer for railway rolling stock, IEEE Trans. Appl. Supercond., Vol.17, No.2, 1927-1930, 2007.

[9] H. J. Kim, J. H. Kim, J. W. Cho, K. D. Sim, S. Kim, S. S. Oh, D. S. Kwag, H. J. Kim, J. H. Bae, and K. C. Seong, AC loss characteristics of Bi-2223 HTS tapes under bending, Physica C, Vol.445-448, 768-771, 2006.

[10] S. P. Ashworth, and M. Suenaga, Local calorimetry to measure ac loss in HTS conductors cryogenics, Vol.41, 77-89, 2001

[11] M. Yagi, S. Tanaka, S. Mukoyama, M. Mimura, H. Kimura, S. Torii, S. Akita, and A. Kikuchi, Measurement of AC losses in an HTS conductor by calorimetric method, Physica C, Vol.392-396, 1124-1128, 2003.

[12] H. Z. Huang, C. J. Zhang, and S. J. Yan, Calorimetric measurement of AC losses of superconducting magnet, Acta Physica Temperature Humilis Sinica, Vol.3, No.1, 42-46, 1981.

[13] Z. Janu, J. Wild, P. Repa, Z. Jelinek, F. Zizek, L. Peksa, F. Soukup, and R. Tichy, Experimental setup for precise measurement of losses in high-temperature superconducting transformer, Cryogenics, Vol.46, 759-761, 2006.

[14] L. Ren, Y. J. Tang, Y. Hu, W. Cai, X. S. Chen, J. Shi, and Y. H. Zhang, Study on service test procedure for superconducting transformers, Cryo. \& Supercond., Vol.35, No.5, 403-408, 2007.

[15] N. Nanato, Y. Tsumiyama, S.B. Kim, S. Murase, K. C. Seong, and H. J. Kim, Development of quench protection system for HTS coils by active power method, Physica C, Vol.463-465, 1281-1284, 2007.

[16] Y. Hwang, S. Lee, W. Kim, K. Choi, and H. Lee, Detection of quench in HTS transformer windings, Physica C, Vol.463-465, 1229-1232, 2007. 